\title{
CONSTRUCTION OF THE OPTIMAL SET OF QUADRATURE RULES IN THE SENSE OF BORGES*
}

\author{
ALEKSANDAR N. JOVANOVIĆ ${ }^{\dagger}$, MARIJA P. STANIĆ ${ }^{\dagger}$, AND TATJANA V. TOMOVIĆ ${ }^{\dagger}$ \\ Dedicated to Walter Gautschi on the occasion of his 90th birthday
}

\begin{abstract}
In this paper we give a numerical method for the construction of an optimal set of quadrature rules in the sense of Borges [Numer. Math., 67 (1994), pp. 271-288] for definite integrals with the same integrand and interval of integration but with different weight functions related to an arbitrary multi-index. We present a numerical method for the construction of an optimal set of quadrature rules in the sense of Borges for four weight functions and explain how to perform an analogous construction for an arbitrary number of weight functions.
\end{abstract}

Key words. multi-index, optimal set of quadrature rules, multiple orthogonal polynomials

AMS subject classifications. 65D32, 42C05

1. Introduction. One generalization of the well-known orthogonal polynomials are multiple orthogonal polynomials, also known as poly-orthogonal polynomials or HermitePadé polynomials. They appeared in [1] to obtain a Hermite-Padé approximation. Because of the wide range of applications, in rational approximation, number theory, random matrices, integrable systems, and geometric function theory, multiple orthogonal polynomials have been extensively studied during the past few decades; see, e.g., [2, 3, 4, 12, 15, 17, 18, 21, 23].

Multiple orthogonal polynomials are polynomials of one variable that satisfy orthogonality conditions with respect to a certain number of weight functions. Regarding the weight functions, there are two kinds of systems: Angelesco systems and AT systems. For Angelesco systems, the weight functions are supported on disjoint intervals (see $[11,12,13])$. On the other hand, for AT systems all weight functions are supported on the same interval. They are extensively investigated by many authors (see $[3,4,6,16,22])$. In this paper we will consider only AT systems.

Now, we give some basic facts and properties of multiple orthogonal polynomials which are necessary for the rest of this paper. Let us denote the set of positive integers by $\mathbb{N}$. Let $\mathbf{n}=\left(n_{1}, n_{2}, \ldots, n_{r}\right)$ be a multi-index, i.e., a vector of $r$ nonnegative integers, with length $|\mathbf{n}|=n_{1}+n_{2}+\cdots+n_{r}$, where $r \in \mathbb{N}$. Let $W=\left(w_{1}, w_{2}, \ldots, w_{r}\right)$ be a vector of weight functions on the real line so that the support of each $w_{i}, i=1,2, \ldots, r$, is a subset of an interval $E_{i}, i=1,2, \ldots, r$. The set of weight functions forms an AT system for the multi-index $\mathbf{n}$ if all weight functions are supported on the same interval $E$ and the set $\left\{x^{\nu} w_{j}(x): \nu=0,1, \ldots, n_{j}-1, j=1,2, \ldots, r\right\}$ forms a Chebyshev system on $E$.

There are two types of multiple orthogonal polynomials. Type I multiple orthogonal polynomials are less important for this paper, so we refer the reader to the book [12, Chapter 23] for definitions and basic properties of these polynomials.

The type II multiple orthogonal polynomial with respect to $(W, \mathbf{n})$ is the monic polynomial $P_{\mathbf{n}}(x)$ of degree $|\mathbf{n}|$ that satisfies the following orthogonality conditions:

$$
\int_{E} P_{\mathbf{n}}(x) x^{k} w_{j}(x) \mathrm{d} x=0, \quad k=0,1, \ldots, n_{j}-1, \quad j=1,2, \ldots, r .
$$

* Received October 29, 2018. Accepted December 6, 2018. Published online on January 16, 2019. Recommended by G.V. Milovanović. The authors were supported in part by the Serbian Ministry of Education, Science and Technological Development (grant numbers \#174015)

${ }^{\dagger}$ University of Kragujevac, Faculty of Science, Radoja Domanovića 12, 34000 Kragujevac, Serbia ( $\{$ aca.jovanovic, stanicm, tomovict\}@kg.ac.rs). 
By using (1.1) we obtain a system of $|\mathbf{n}|$ linear equations for the $|\mathbf{n}|$ unknown coefficients of the monic polynomial $P_{\mathbf{n}}(x)=\sum_{k=0}^{|\mathbf{n}|} a_{k, \mathbf{n}} x^{k}, a_{|\mathbf{n}|, \mathbf{n}}=1$. If the system (1.1) has a unique solution, then the multi-index $\mathbf{n}$ is normal for type II, and the polynomial $P_{\mathbf{n}}(x)$ is unique. If all multi-indices are normal, then we have a perfect system. AT systems are perfect systems.

For the (monic) orthogonal polynomials with respect to each weight function $w_{j}$, $j=1,2, \ldots, r$, the recurrence relation

$$
x P_{n}\left(x ; w_{j}\right)=P_{n+1}\left(x ; w_{j}\right)+b_{n}\left(w_{j}\right) P_{n}\left(x ; w_{j}\right)+a_{n}^{2}\left(w_{j}\right) P_{n-1}\left(x ; w_{j}\right)
$$

and the corresponding coefficients will be called marginal (see [10]).

A multi-index of the form

$$
\mathbf{d}(n)=(\underbrace{\ell+1, \ell+1, \ldots, \ell+1}_{j \text { times }}, \underbrace{\ell, \ell, \ldots, \ell}_{r-j \text { times }}),
$$

where $n=r \ell+j, \ell=[n / r], 0 \leq j \leq r$, is called nearly-diagonal. The corresponding type II multiple orthogonal polynomial $P_{\mathbf{d}(n)}(x)$ satisfies the following nearly-diagonal recurrence relation

$$
x P_{\mathbf{d}(n)}(x)=P_{\mathbf{d}(n+1)}(x)+\sum_{i=0}^{r} \alpha_{n, r-i} P_{\mathbf{d}(n-i)}(x), \quad n \geq 0,
$$

with the initial conditions $P_{\mathbf{d}(0)}(x)=1$ and $P_{\mathbf{d}(i)}(x)=0$, for $i=-1,-2, \ldots,-r$ (see $[14,23])$.

Assuming that all multi-indices are normal, the following nearest neighbor recurrence relations

$$
x P_{\mathbf{n}}(x)=P_{\mathbf{n}+\mathbf{e}_{k}}(x)+b_{\mathbf{n}}^{(k)} P_{\mathbf{n}}(x)+\sum_{j=1}^{r} a_{\mathbf{n}}^{(j)} P_{\mathbf{n}-\mathbf{e}_{j}}(x), \quad k=1,2, \ldots, r,
$$

hold with the initial conditions $P_{\mathbf{0}}(x)=1$ and $P_{-\mathbf{e}_{j}}(x)=0, j=1,2, \ldots, r$, where $\mathbf{e}_{j}$ is the $j$ th standard unit vector with 1 at the $j$ th entry and the vectors $\left(a_{\mathbf{n}}^{(1)}, a_{\mathbf{n}}^{(2)}, \ldots, a_{\mathbf{n}}^{(r)}\right)$ and $\left(b_{\mathbf{n}}^{(1)}, b_{\mathbf{n}}^{(2)}, \ldots, b_{\mathbf{n}}^{(r)}\right)$ are the recurrence coefficients (see [12, 22]).

The important property of the zeros of type II multiple orthogonal polynomials is given by the following theorem.

THEOREM 1.1 ([20]). Suppose that $\mathbf{n}$ is a multi-index and that $W=\left(w_{1}, w_{2}, \ldots, w_{r}\right)$ is an AT system of weight functions on an interval $E$ for the multi-index $\mathbf{n}$. The type II multiple orthogonal polynomial $P_{\mathbf{n}}(x)$ with respect to $(W, \mathbf{n})$ has exactly $|\mathbf{n}|$ simple zeros on $E$.

The paper is organized as follows. In Section 2 a method for constructing type II multiple orthogonal polynomials is given. The case of four weight functions is presented in detail, but the method for arbitrary $r$ is described as well. Section 3 is devoted to the application in numerical integration and to the corresponding methods for calculating the nodes and weight coefficients of the optimal set of quadrature rules. The numerical construction of the mentioned quadrature rules for $r=4$ is presented in Section 3.1, where also one numerical example is given.

2. Constructing type II multiple orthogonal polynomials. In this section we present a method for constructing type II multiple orthogonal polynomial $P_{\left(n_{1}, n_{2}, n_{3}, n_{4}\right)}(x)$ corresponding to a multi-index $\mathbf{n}=\left(n_{1}, n_{2}, n_{3}, n_{4}\right) \in \mathbb{N}^{4}$ and with respect to weight functions $W=\left(w_{1}, w_{2}, w_{3}, w_{4}\right)$. At the end we explain how this method could be extended to the 
general case. For $r=4$, the nearest neighbor recurrence relations (1.4) have the following form:

$$
\begin{aligned}
x P_{\mathbf{n}}(x)= & P_{\mathbf{n}+\mathbf{e}_{1}}(x)+b_{\mathbf{n}}^{(1)} P_{\mathbf{n}}(x)+a_{\mathbf{n}}^{(1)} P_{\mathbf{n}-\mathbf{e}_{1}}(x)+a_{\mathbf{n}}^{(2)} P_{\mathbf{n}-\mathbf{e}_{2}}(x) \\
& +a_{\mathbf{n}}^{(3)} P_{\mathbf{n}-\mathbf{e}_{3}}(x)+a_{\mathbf{n}}^{(4)} P_{\mathbf{n}-\mathbf{e}_{4}}(x), \\
x P_{\mathbf{n}}(x)= & P_{\mathbf{n}+\mathbf{e}_{2}}(x)+b_{\mathbf{n}}^{(2)} P_{\mathbf{n}}(x)+a_{\mathbf{n}}^{(1)} P_{\mathbf{n}-\mathbf{e}_{1}}(x)+a_{\mathbf{n}}^{(2)} P_{\mathbf{n}-\mathbf{e}_{2}}(x) \\
& +a_{\mathbf{n}}^{(3)} P_{\mathbf{n}-\mathbf{e}_{3}}(x)+a_{\mathbf{n}}^{(4)} P_{\mathbf{n}-\mathbf{e}_{4}}(x), \\
x P_{\mathbf{n}}(x)= & P_{\mathbf{n}+\mathbf{e}_{3}}(x)+b_{\mathbf{n}}^{(3)} P_{\mathbf{n}}(x)+a_{\mathbf{n}}^{(1)} P_{\mathbf{n}-\mathbf{e}_{1}}(x)+a_{\mathbf{n}}^{(2)} P_{\mathbf{n}-\mathbf{e}_{2}}(x) \\
& +a_{\mathbf{n}}^{(3)} P_{\mathbf{n}-\mathbf{e}_{3}}(x)+a_{\mathbf{n}}^{(4)} P_{\mathbf{n}-\mathbf{e}_{4}}(x), \\
x P_{\mathbf{n}}(x)= & P_{\mathbf{n}+\mathbf{e}_{4}}(x)+b_{\mathbf{n}}^{(4)} P_{\mathbf{n}}(x)+a_{\mathbf{n}}^{(1)} P_{\mathbf{n}-\mathbf{e}_{1}}(x)+a_{\mathbf{n}}^{(2)} P_{\mathbf{n}-\mathbf{e}_{2}}(x) \\
& +a_{\mathbf{n}}^{(3)} P_{\mathbf{n}-\mathbf{e}_{3}}(x)+a_{\mathbf{n}}^{(4)} P_{\mathbf{n}-\mathbf{e}_{4}}(x) .
\end{aligned}
$$

Let us point out that the coefficients $a_{\mathbf{n}}^{(j)}, j=1,2, \ldots, r$, in (1.4) do not depend on $k$, and because of that we have the same coefficients $a_{\mathbf{n}}^{(1)}, a_{\mathbf{n}}^{(2)}, a_{\mathbf{n}}^{(3)}$, and $a_{\mathbf{n}}^{(4)}$ in (2.1)-(2.4).

The following three lemmas could be easily proved by using mathematical induction (similarly as in [19]).

LEMMA 2.1. Let $\left(n_{1}, n_{2}, n_{3}, n_{4}\right) \in \mathbb{N}^{4}$ be a multi-index and $P_{\left(n_{1}, n_{2}, n_{3}, n_{4}\right)}(x)$ the type II multiple orthogonal polynomial with respect to $W$. Then

$$
\begin{aligned}
P_{\mathbf{n}+\mathbf{e}_{2}}(x)=P_{\mathbf{n}+\mathbf{e}_{1}}(x) & +\sum_{k=0}^{n_{2}-1} P_{\left(n_{1}+1, k, n_{3}, n_{4}\right)}(x) \prod_{i=k+1}^{n_{2}}\left(b_{\left(n_{1}, i, n_{3}, n_{4}\right)}^{(1)}-b_{\left(n_{1}, i, n_{3}, n_{4}\right)}^{(2)}\right) \\
& +P_{\left(n_{1}, 0, n_{3}, n_{4}\right)}(x) \prod_{i=0}^{n_{2}}\left(b_{\left(n_{1}, i, n_{3}, n_{4}\right)}^{(1)}-b_{\left(n_{1}, i, n_{3}, n_{4}\right)}^{(2)}\right)
\end{aligned}
$$

holds.

LEMMA 2.2. Let $\left(n_{1}, n_{2}, n_{3}, n_{4}\right) \in \mathbb{N}^{4}$ be a multi-index and $P_{\left(n_{1}, n_{2}, n_{3}, n_{4}\right)}(x)$ the type II multiple orthogonal polynomial with respect to $W$. Then

$$
\begin{array}{r}
P_{\mathbf{n}+\mathbf{e}_{3}}(x)=P_{\mathbf{n}+\mathbf{e}_{\ell}}(x)+\sum_{k=0}^{n_{3}-1} P_{\left(n_{1}, n_{2}, k, n_{4}\right)+\mathbf{e}_{\ell}}(x) \prod_{i=k+1}^{n_{3}}\left(b_{\left(n_{1}, n_{2}, i, n_{4}\right)}^{(\ell)}-b_{\left(n_{1}, n_{2}, i, n_{4}\right)}^{(3)}\right) \\
+P_{\left(n_{1}, n_{2}, 0, n_{4}\right)}(x) \prod_{i=0}^{n_{3}}\left(b_{\left(n_{1}, n_{2}, i, n_{4}\right)}^{(\ell)}-b_{\left(n_{1}, n_{2}, i, n_{4}\right)}^{(3)}\right), \quad \ell=1,2,
\end{array}
$$

holds.

LEMMA 2.3. Let $\left(n_{1}, n_{2}, n_{3}, n_{4}\right) \in \mathbb{N}^{4}$ be a multi-index and $P_{\left(n_{1}, n_{2}, n_{3}, n_{4}\right)}(x)$ the type II multiple orthogonal polynomial with respect to $W$. Then

$$
\begin{array}{r}
P_{\mathbf{n}+\mathbf{e}_{4}}(x)=P_{\mathbf{n}+\mathbf{e}_{\ell}}(x)+\sum_{k=0}^{n_{4}-1} P_{\left(n_{1}, n_{2}, n_{3}, k\right)+\mathbf{e}_{\ell}}(x) \prod_{i=k+1}^{n_{4}}\left(b_{\left(n_{1}, n_{2}, n_{3}, i\right)}^{(\ell)}-b_{\left(n_{1}, n_{2}, n_{3}, i\right)}^{(4)}\right) \\
+P_{\left(n_{1}, n_{2}, n_{3}, 0\right)}(x) \prod_{i=0}^{n_{4}}\left(\left(b_{\left(n_{1}, n_{2}, n_{3}, i\right)}^{(\ell)}-b_{\left(n_{1}, n_{2}, n_{3}, i\right)}^{(4)}\right), \quad \ell=1,2,3\right.
\end{array}
$$

holds. 
Here we present one way of constructing the polynomial $P_{(n, m, p, q)}(x)$. Setting the indices $n_{1}=0,1, \ldots, n-1$ and $n_{2}=n_{3}=n_{4}=0$ in $(2.1)$, we get

$$
\begin{aligned}
x P_{(0,0,0,0)}(x) & =P_{(1,0,0,0)}(x)+b_{(0,0,0,0)}^{(1)} P_{(0,0,0,0)}(x), \\
x P_{(1,0,0,0)}(x) & =P_{(2,0,0,0)}(x)+b_{(1,0,0,0)}^{(1)} P_{(1,0,0,0)}(x)+a_{(1,0,0,0)}^{(1)} P_{(0,0,0,0)}(x), \\
x P_{(2,0,0,0)}(x) & =P_{(3,0,0,0)}(x)+b_{(2,0,0,0)}^{(1)} P_{(2,0,0,0)}(x)+a_{(2,0,0,0)}^{(1)} P_{(1,0,0,0)}(x), \\
& \vdots \\
x P_{(n-1,0,0,0)}(x) & =P_{(n, 0,0,0)}(x)+b_{(n-1,0,0,0)}^{(1)} P_{(n-1,0,0,0)}(x)+a_{(n-1,0,0,0)}^{(1)} P_{(n-2,0,0,0)}(x) .
\end{aligned}
$$

Now by applying (2.2) with $n_{1}=n, n_{2}=0,1$, and $n_{3}=n_{4}=0$, we obtain

$$
\begin{aligned}
x P_{(n, 0,0,0)}(x)=P_{(n, 1,0,0)}(x) & +b_{(n, 0,0,0)}^{(2)} P_{(n, 0,0,0)}(x)+a_{(n, 0,0,0)}^{(1)} P_{(n-1,0,0,0)}(x), \\
x P_{(n, 1,0,0)}(x)=P_{(n, 2,0,0)}(x) & +b_{(n, 1,0,0)}^{(2)} P_{(n, 1,0,0)}(x)+a_{(n, 1,0,0)}^{(1)} P_{(n-1,1,0,0)}(x) \\
& +a_{(n, 1,0,0)}^{(2)} P_{(n, 0,0,0)}(x) .
\end{aligned}
$$

By using (2.5), for $\left(n_{1}, n_{2}, n_{3}, n_{4}\right)=(n-1,0,0,0)$, we have

$$
P_{(n-1,1,0,0)}(x)=P_{(n, 0,0,0)}(x)+P_{(n-1,0,0,0)}(x)\left(b_{(n-1,0,0,0)}^{(1)}-b_{(n-1,0,0,0)}^{(2)}\right),
$$

and hence

$$
\begin{aligned}
x P_{(n, 1,0,0)}(x)=P_{(n, 2,0,0)}(x) & +b_{(n, 1,0,0)}^{(2)} P_{(n, 1,0,0)}(x) \\
& +\left(a_{(n, 1,0,0)}^{(1)}+a_{(n, 1,0,0)}^{(2)}\right) P_{(n, 0,0,0)}(x) \\
& +a_{(n, 1,0,0)}^{(1)}\left(b_{(n-1,0,0,0)}^{(1)}-b_{(n-1,0,0,0)}^{(2)}\right) P_{(n-1,0,0,0)}(x) .
\end{aligned}
$$

In the same way, by using (2.2) and (2.5), we obtain

$$
\begin{aligned}
& x P_{(n, 2,0,0)}(x)= P_{(n, 3,0,0)}(x)+b_{(n, 2,0,0)}^{(2)} P_{(n, 2,0,0)}(x) \\
&+\left(a_{(n, 2,0,0)}^{(1)}+a_{(n, 2,0,0)}^{(2)}\right) P_{(n, 1,0,0)}(x) \\
&+a_{(n, 2,0,0)}^{(1)}\left(b_{(n-1,1,0,0)}^{(1)}-b_{(n-1,1,0,0)}^{(2)}\right) P_{(n, 0,0,0)}(x) \\
&+a_{(n, 2,0,0)}^{(1)} \prod_{i=0}^{1}\left(b_{(n-1, i, 0,0)}^{(1)}-b_{(n-1, i, 0,0)}^{(2)}\right) P_{(n-1,0,0,0)}(x) \\
& \vdots \quad \\
& x P_{(n, m-1,0,0)}(x)=P_{(n, m, 0,0)}(x)+b_{(n, m-1,0,0)}^{(2)} P_{(n, m-1,0,0)}(x) \\
& \quad+\left(a_{(n, m-1,0,0)}^{(1)}+a_{(n, m-1,0,0)}^{(2)}\right) P_{(n, m-2,0,0)}(x) \\
&+a_{(n, m-1,0,0)}^{(1)} \sum_{k=0}^{m-3} P_{(n, k, 0,0)}(x) \prod_{i=k+1}^{m-2}\left(b_{(n-1, i, 0,0)}^{(1)}-b_{(n-1, i, 0,0)}^{(2)}\right) \\
&+a_{(n, m-1,0,0)}^{(1)} \prod_{i=0}^{m-2}\left(b_{(n-1, i, 0,0)}^{(1)}-b_{(n-1, i, 0,0)}^{(2)}\right) P_{(n-1,0,0,0)}(x) .
\end{aligned}
$$


Now by applying (2.3) with $\left(n_{1}, n_{2}, n_{3}, n_{4}\right)=(n, m, 0,0)$, we obtain

$$
\begin{aligned}
x P_{(n, m, 0,0)}(x)=P_{(n, m, 1,0)}(x) & +b_{(n, m, 0,0)}^{(3)} P_{(n, m, 0,0)}(x)+a_{(n, m, 0,0)}^{(1)} P_{(n-1, m, 0,0)}(x) \\
& +a_{(n, m, 0,0)}^{(2)} P_{(n, m-1,0,0)}(x) .
\end{aligned}
$$

By using (2.5), for $\left(n_{1}, n_{2}, n_{3}, n_{4}\right)=(n-1, m-1,0,0)$, we have

$$
\begin{aligned}
& P_{(n-1, m, 0,0)}(x)=P_{(n, m-1,0,0)}(x) \\
& \quad+\sum_{k=0}^{m-2} P_{(n, k, 0,0)}(x) \prod_{i=k+1}^{m-1}\left(b_{(n-1, i, 0,0)}^{(1)}-b_{(n-1, i, 0,0)}^{(2)}\right) \\
& +\prod_{i=0}^{m-1}\left(b_{(n-1, i, 0,0)}^{(1)}-b_{(n-1, i, 0,0)}^{(2)}\right) P_{(n-1,0,0,0)},
\end{aligned}
$$

and hence

$$
\begin{aligned}
x P_{(n, m, 0,0)}(x)=P_{(n, m, 1,0)}(x)+b_{(n, m, 0,0)}^{(3)} P_{(n, m, 0,0)}(x) \\
+\left(a_{(n, m, 0,0)}^{(1)}+a_{(n, m, 0,0)}^{(2)}\right) P_{(n, m-1,0,0)}(x) \\
+a_{(n, m, 0,0)}^{(1)} \sum_{k=0}^{m-2} P_{(n, k, 0,0)}(x) \prod_{i=k+1}^{m-1}\left(b_{(n-1, i, 0,0)}^{(1)}-b_{(n-1, i, 0,0)}^{(2)}\right) \\
+a_{(n, m, 0,0)}^{(1)} \prod_{i=0}^{m-1}\left(b_{(n-1, i, 0,0)}^{(1)}-b_{(n-1, i, 0,0)}^{(2)}\right) P_{(n-1,0,0,0)}(x) .
\end{aligned}
$$

In a similar way, by applying (2.3) with $\left(n_{1}, n_{2}, n_{3}, n_{4}\right)=(n, m, 1,0)$, we obtain

$$
\begin{aligned}
x P_{(n, m, 1,0)}(x)=P_{(n, m, 2,0)}(x) & +b_{(n, m, 1,0)}^{(3)} P_{(n, m, 1,0)}(x)+a_{(n, m, 1,0)}^{(1)} P_{(n-1, m, 1,0)}(x) \\
& +a_{(n, m, 1,0)}^{(2)} P_{(n, m-1,1,0)}(x)+a_{(n, m, 1,0)}^{(3)} P_{(n, m, 0,0)}(x) .
\end{aligned}
$$

By using (2.6), where $\ell=1$, for the multi-index $\left(n_{1}, n_{2}, n_{3}, n_{4}\right)=(n-1, m, 0,0)$ and (2.5), for the multi-index $\left(n_{1}, n_{2}, n_{3}, n_{4}\right)=(n-1, m-1,0,0)$ (or (2.8)), we have

$$
\begin{aligned}
P_{(n-1, m, 1,0)}(x)= & P_{(n, m, 0,0)}(x)+\left(b_{(n-1, m, 0,0)}^{(1)}-b_{(n-1, m, 0,0)}^{(3)}\right) P_{(n-1, m, 0,0)}(x) \\
= & P_{(n, m, 0,0)}(x)+\left(b_{(n-1, m, 0,0)}^{(1)}-b_{(n-1, m, 0,0)}^{(3)}\right) P_{(n, m-1,0,0)}(x) \\
+ & \left(b_{(n-1, m, 0,0)}^{(1)}-b_{(n-1, m, 0,0)}^{(3)}\right) \\
& \times \sum_{k=0}^{m-2} P_{(n, k, 0,0)}(x) \prod_{i=k+1}^{m-1}\left(b_{(n-1, i, 0,0)}^{(1)}-b_{(n-1, i, 0,0)}^{(2)}\right) \\
+ & \left(b_{(n-1, m, 0,0)}^{(1)}-b_{(n-1, m, 0,0)}^{(3)}\right) \prod_{i=0}^{m-1}\left(b_{(n-1, i, 0,0)}^{(1)}-b_{(n-1, i, 0,0)}^{(2)}\right) \\
& \times P_{(n-1,0,0,0)}(x),
\end{aligned}
$$

and by using (2.6), where $\ell=2$, for $\left(n_{1}, n_{2}, n_{3}, n_{4}\right)=(n, m-1,0,0)$, we have

$$
P_{(n, m-1,1,0)}(x)=P_{(n, m, 0,0)}(x)+\left(b_{(n, m-1,0,0)}^{(2)}-b_{(n, m-1,0,0)}^{(3)}\right) P_{(n, m-1,0,0)}(x),
$$


and hence

$$
\begin{aligned}
x P_{(n, m, 1,0)}(x)= & P_{(n, m, 2,0)}(x)+b_{(n, m, 1,0)}^{(3)} P_{(n, m, 1,0)}(x) \\
+ & \left(a_{(n, m, 1,0)}^{(1)}+a_{(n, m, 1,0)}^{(2)}+a_{(n, m, 1,0)}^{(3)}\right) P_{(n, m, 0,0)}(x) \\
+ & \left(a_{(n, m, 1,0)}^{(1)}\left(b_{(n-1, m, 0,0)}^{(1)}-b_{(n-1, m, 0,0)}^{(3)}\right)\right. \\
& \left.+a_{(n, m, 1,0)}^{(2)}\left(b_{(n, m-1,0,0)}^{(2)}-b_{(n, m-1,0,0)}^{(3)}\right)\right) P_{(n, m-1,0,0)}(x) \\
+ & a_{(n, m, 1,0)}^{(1)}\left(b_{(n-1, m, 0,0)}^{(1)}-b_{(n-1, m, 0,0)}^{(3)}\right) \\
& \quad \times \sum_{k=0}^{m-2} P_{(n, k, 0,0)}(x) \prod_{i=k+1}^{m-1}\left(b_{(n-1, i, 0,0)}^{(1)}-b_{(n-1, i, 0,0)}^{(2)}\right) \\
+ & a_{(n, m, 1,0)}^{(1)}\left(b_{(n-1, m, 0,0)}^{(1)}-b_{(n-1, m, 0,0)}^{(3)}\right) \\
& \quad \times \prod_{i=0}^{m-1}\left(b_{(n-1, i, 0,0)}^{(1)}-b_{(n-1, i, 0,0)}^{(2)}\right) P_{(n-1,0,0,0)}(x) .
\end{aligned}
$$

Continuing in this manner, by using (2.3) for $\left(n_{1}, n_{2}, n_{3}, n_{4}\right)=(n, m, p-1,0)$, (2.6), where $\ell=1$, for $\left(n_{1}, n_{2}, n_{3}, n_{4}\right)=(n-1, m, p-2,0)$, (2.8), and (2.6), where $\ell=2$, for $\left(n_{1}, n_{2}, n_{3}, n_{4}\right)=(n, m-1, p-2,0)$, we obtain

$$
\begin{aligned}
& x P_{(n, m, p-1,0)}(x)=P_{(n, m, p, 0)}(x)+b_{(n, m, p-1,0)}^{(3)} P_{(n, m, p-1,0)}(x) \\
& +\left(a_{(n, m, p-1,0)}^{(1)}+a_{(n, m, p-1,0)}^{(2)}+a_{(n, m, p-1,0)}^{(3)}\right) P_{(n, m, p-2,0)}(x) \\
& +\sum_{k=0}^{p-3} P_{(n, m, k, 0)}(x)\left(a_{(n, m, p-1,0)}^{(1)} \prod_{i=k+1}^{p-2}\left(b_{(n-1, m, i, 0)}^{(1)}-b_{(n-1, m, i, 0)}^{(3)}\right)\right. \\
& \left.+a_{(n, m, p-1,0)}^{(2)} \prod_{i=k+1}^{p-2}\left(b_{(n, m-1, i, 0)}^{(2)}-b_{(n, m-1, i, 0)}^{(3)}\right)\right) \\
& +P_{(n, m-1,0,0)}(x)\left(a_{(n, m, p-1,0)}^{(1)} \prod_{i=0}^{p-2}\left(b_{(n-1, m, i, 0)}^{(1)}-b_{(n-1, m, i, 0)}^{(3)}\right)\right. \\
& \left.+a_{(n, m, p-1,0)}^{(2)} \prod_{i=0}^{p-2}\left(b_{(n, m-1, i, 0)}^{(2)}-b_{(n, m-1, i, 0)}^{(3)}\right)\right) \\
& +a_{(n, m, p-1,0)}^{(1)} \prod_{i=0}^{p-2}\left(b_{(n-1, m, i, 0)}^{(1)}-b_{(n-1, m, i, 0)}^{(3)}\right) \\
& \times \sum_{k=0}^{m-2} P_{(n, k, 0,0)}(x) \prod_{i=k+1}^{m-1}\left(b_{(n-1, i, 0,0)}^{(1)}-b_{(n-1, i, 0,0)}^{(2)}\right) \\
& +P_{(n-1,0,0)}(x) a_{(n, m, p-1,0)}^{(1)} \prod_{i=0}^{p-2}\left(b_{(n-1, m, i, 0)}^{(1)}-b_{(n-1, m, i, 0)}^{(3)}\right) \\
& \times \prod_{i=0}^{m-1}\left(b_{(n-1, i, 0,0)}^{(1)}-b_{(n-1, i, 0,0)}^{(2)}\right) .
\end{aligned}
$$


Now by applying (2.4) with $\left(n_{1}, n_{2}, n_{3}, n_{4}\right)=(n, m, p, 0)$, we obtain

$$
\begin{aligned}
x P_{(n, m, p, 0)}(x)=P_{(n, m, p, 1)}(x) & +b_{(n, m, p, 0)}^{(4)} P_{(n, m, p, 0)}(x)+a_{(n, m, p, 0)}^{(1)} P_{(n-1, m, p, 0)}(x) \\
& +a_{(n, m, p, 0)}^{(2)} P_{(n, m-1, p, 0)}(x)+a_{(n, m, p, 0)}^{(3)} P_{(n, m, p-1,0)}(x) .
\end{aligned}
$$

By using (2.6), where $\ell=1$, for $\left(n_{1}, n_{2}, n_{3}, n_{4}\right)=(n-1, m, p-1,0)$, and (2.8) (which is equal to (2.5) for $\left.\left(n_{1}, n_{2}, n_{3}, n_{4}\right)=(n-1, m-1,0,0)\right)$, we have

$$
\begin{aligned}
P_{(n-1, m, p, 0)}(x)= & P_{(n, m, p-1,0)}(x)+\sum_{k=0}^{p-2} P_{(n, m, k, 0)}(x) \prod_{i=k+1}^{p-1}\left(b_{(n-1, m, i, 0)}^{(1)}-b_{(n-1, m, i, 0)}^{(3)}\right) \\
& +P_{(n-1, m, 0,0)}(x) \prod_{i=0}^{p-1}\left(b_{(n-1, m, i, 0)}^{(1)}-b_{(n-1, m, i, 0)}^{(3)}\right) \\
& +\sum_{k=0}^{m-2} P_{(n, k, 0,0)}(x) \prod_{i=k+1}^{m-1}\left(b_{(n-1, i, 0,0)}^{(1)}-b_{(n-1, i, 0,0)}^{(2)}\right) \\
& \times \prod_{(n-1,0,0,0)}(x) \prod_{i=0}^{m-1}\left(b_{(n-1, m, i, 0)}^{(1)}-b_{(n-1, m, i, 0)}^{(3)}\right) \\
& \times \prod_{i=0}^{p-1}\left(b_{(n-1, i, m, i, 0)}^{(1)}-b_{(n-1, m, i, 0)}^{(3)}-b_{(n-1, i, 0,0)}^{(1)}\right)
\end{aligned}
$$

and by using (2.6), where $\ell=2$, for $\left(n_{1}, n_{2}, n_{3}, n_{4}\right)=(n, m-1, p-1,0)$, we have

$$
\begin{aligned}
P_{(n, m-1, p, 0)}(x)= & P_{(n, m, p-1,0)}(x)+\sum_{k=0}^{p-2} P_{(n, m, k, 0)}(x) \prod_{i=k+1}^{p-1}\left(b_{(n, m-1, i, 0)}^{(2)}-b_{(n, m-1, i, 0)}^{(3)}\right) \\
& +P_{(n, m-1,0,0)}(x) \prod_{i=0}^{p-1}\left(b_{(n, m-1, i, 0)}^{(2)}-b_{(n, m-1, i, 0)}^{(3)}\right) .
\end{aligned}
$$

Hence,

$$
\begin{aligned}
x P_{(n, m, p, 0)}(x)= & P_{(n, m, p, 1)}(x)+b_{(n, m, p, 0)}^{(4)} P_{(n, m, p, 0)}(x) \\
& \left.+a_{(n, m, p, 0)}^{(1)}+a_{(n, m, p, 0)}^{(2)}+a_{(n, m, p, 0)}^{(3)}\right) P_{(n, m, p-1,0)}(x) \\
& +\sum_{k=0}^{p-2} P_{(n, m, k, 0)}(x)\left(a_{(n, m, p, 0)}^{(1)} \prod_{i=k+1}^{p-1}\left(b_{(n-1, m, i, 0)}^{(1)}-b_{(n-1, m, i, 0)}^{(3)}\right)\right. \\
& \left.\quad+a_{(n, m, p, 0)}^{(2)} \prod_{i=k+1}^{p-1}\left(b_{(n, m-1, i, 0)}^{(2)}-b_{(n, m-1, i, 0)}^{(3)}\right)\right) \\
& +P_{(n, m-1,0,0)}(x)\left(a_{(n, m, p, 0)}^{(1)} \prod_{i=0}^{p-1}\left(b_{(n-1, m, i, 0)}^{(1)}-b_{(n-1, m, i, 0)}^{(3)}\right)\right.
\end{aligned}
$$




$$
\begin{array}{r}
\left.+a_{(n, m, p, 0)}^{(2)} \prod_{i=0}^{p-1}\left(b_{(n, m-1, i, 0)}^{(2)}-b_{(n, m-1, i, 0)}^{(3)}\right)\right) \\
+a_{(n, m, p, 0)}^{(1)} \prod_{i=0}^{p-1}\left(b_{(n-1, m, i, 0)}^{(1)}-b_{(n-1, m, i, 0)}^{(3)}\right) \\
\times \sum_{k=0}^{m-2} P_{(n, k, 0,0)}(x) \prod_{i=k+1}^{m-1}\left(b_{(n-1, i, 0,0)}^{(1)}-b_{(n-1, i, 0,0)}^{(2)}\right) \\
+P_{(n-1,0,0)}(x) a_{(n, m, p, 0)}^{(1)} \prod_{i=0}^{p-1}\left(b_{(n-1, m, i, 0)}^{(1)}-b_{(n-1, m, i, 0)}^{(3)}\right) \\
\times \prod_{i=0}^{m-1}\left(b_{(n-1, i, 0,0)}^{(1)}-b_{(n-1, i, 0,0)}^{(2)}\right) .
\end{array}
$$

In a similar way by applying (2.4) with $\left(n_{1}, n_{2}, n_{3}, n_{4}\right)=(n, m, p, 1)$, we obtain

$$
\begin{gathered}
x P_{(n, m, p, 1)}(x)=P_{(n, m, p, 2)}(x)+b_{(n, m, p, 1)}^{(4)} P_{(n, m, p, 1)}(x)+a_{(n, m, p, 1)}^{(1)} P_{(n-1, m, p, 1)}(x) \\
+a_{(n, m, p, 1)}^{(2)} P_{(n, m-1, p, 1)}(x)+a_{(n, m, p, 1)}^{(3)} P_{(n, m, p-1,1)}(x) \\
+a_{(n, m, p, 1)}^{(4)} P_{(n, m, p, 0)}(x) .
\end{gathered}
$$

By using (2.7), where $\ell=1$, for $\left(n_{1}, n_{2}, n_{3}, n_{4}\right)=(n-1, m, p, 0)$, and (2.9), we express $P_{(n-1, m, p, 1)}(x)$. By using (2.7), where $\ell=2$, for $\left(n_{1}, n_{2}, n_{3}, n_{4}\right)=(n, m-1, p, 0)$, and (2.10), we express $P_{(n, m-1, p, 1)}(x)$, and by using (2.7), where $\ell=3$, for $\left(n_{1}, n_{2}, n_{3}, n_{4}\right)=$ $(n, m, p-1,0)$, we express $P_{(n, m, p-1,1)}(x)$. Hence we have

$$
\begin{aligned}
& x P_{(n, m, p, 1)}(x) \\
& =P_{(n, m, p, 2)}(x)+b_{(n, m, p, 1)}^{(4)} P_{(n, m, p, 1)}(x) \\
& +\left(a_{(n, m, p, 1)}^{(1)}+a_{(n, m, p, 1)}^{(2)}+a_{(n, m, p, 1)}^{(3)}\right) P_{(n, m, p, 0)}(x) \\
& +P_{(n, m, p-1,0)}(x)\left(a_{(n, m, p, 1)}^{(1)}\left(b_{(n-1, m, p, 0)}^{(1)}-b_{(n-1, m, p, 0)}^{(4)}\right)\right. \\
& +a_{(n, m, p, 1)}^{(2)}\left(b_{(n, m-1, p, 0)}^{(2)}-b_{(n, m-1, p, 0)}^{(4)}\right) \\
& \left.+a_{(n, m, p, 1)}^{(3)}\left(b_{(n, m, p-1,0)}^{(3)}-b_{(n, m, p-1,0)}^{(4)}\right)\right) \\
& +\sum_{k=0}^{p-2} P_{(n, m, k, 0)}(x)\left(a_{(n, m, p, 1)}^{(1)}\left(b_{(n-1, m, p, 0)}^{(1)}-b_{(n-1, m, p, 0)}^{(4)}\right)\right. \\
& \times \prod_{i=k+1}^{p-1}\left(b_{(n-1, m, i, 0)}^{(1)}-b_{(n-1, m, i, 0)}^{(3)}\right) \\
& +a_{(n, m, p, 1)}^{(2)}\left(b_{(n, m-1, p, 0)}^{(2)}-b_{(n, m-1, p, 0)}^{(4)}\right) \\
& \left.\times \prod_{i=k+1}^{p-1}\left(b_{(n, m-1, i, 0)}^{(2)}-b_{(n, m-1, i, 0)}^{(3)}\right)\right)
\end{aligned}
$$




$$
\begin{aligned}
+P_{(n, m-1,0,0)}(x)\left(a_{(n, m, p, 1)}^{(1)}\left(b_{(n-1, m, p, 0)}^{(1)}-b_{(n-1, m, p, 0)}^{(4)}\right)\right. & \\
\times & \prod_{i=0}^{p-1}\left(b_{(n-1, m, i, 0)}^{(1)}-b_{(n-1, m, i, 0)}^{(3)}\right) \\
+ & a_{(n, m, p, 1)}^{(2)}\left(b_{(n, m-1, p, 0)}^{(2)}-b_{(n, m-1, p, 0)}^{(4)}\right) \\
\times & \left.\prod_{i=0}^{p-1}\left(b_{(n, m-1, i, 0)}^{(2)}-b_{(n, m-1, i, 0)}^{(3)}\right)\right) \\
+a_{(n, m, p, 1)}^{(1)}\left(b_{(n-1, m, p, 0)}^{(1)}-\right. & \left.b_{(n-1, m, p, 0)}^{(4)}\right) \prod_{i=0}^{p-1}\left(b_{(n-1, m, i, 0)}^{(1)}-b_{(n-1, m, i, 0)}^{(3)}\right) \\
\times & \sum_{k=0}^{m-2} P_{(n, k, 0,0)}(x) \prod_{i=k+1}^{m-1}\left(b_{(n-1, i, 0,0)}^{(1)}-b_{(n-1, i, 0,0)}^{(2)}\right) \\
+P_{(n-1,0,0)}(x) a_{(n, m, p, 1)}^{(1)} & \left.b_{(n-1, m, p, 0)}^{(1)}-b_{(n-1, m, p, 0)}^{(4)}\right) \\
& \times \prod_{i=0}^{p-1}\left(b_{(n-1, m, i, 0)}^{(1)}-b_{(n-1, m, i, 0)}^{(3)}\right) \\
& \times \prod_{i=0}^{m-1}\left(b_{(n-1, i, 0,0)}^{(1)}-b_{(n-1, i, 0,0)}^{(2)}\right) .
\end{aligned}
$$

Continuing in this manner, by using (2.4) for $\left(n_{1}, n_{2}, n_{3}, n_{4}\right)=(n, m, p, q-2)$, (2.7), where $\ell=1$, for $\left(n_{1}, n_{2}, n_{3}, n_{4}\right)=(n-1, m, p, q-3)$, (2.9), (2.7), where $\ell=2$, for $\left(n_{1}, n_{2}, n_{3}, n_{4}\right)=(n, m-1, p, q-3),(2.10)$ and $(2.7)$, where $\ell=3$, for $\left(n_{1}, n_{2}, n_{3}, n_{4}\right)=$ $(n, m, p-1, q-3)$, we obtain

$$
\begin{aligned}
& x P_{(n, m, p, q-2)}(x) \\
& =P_{(n, m, p, q-1)}(x)+b_{(n, m, p, q-2)}^{(4)} P_{(n, m, p, q-2)}(x) \\
& \left.+a_{(n, m, p, q-2)}^{(1)}+a_{(n, m, p, q-2)}^{(2)}+a_{(n, m, p, q-2)}^{(3)}+a_{(n, m, p, q-2)}^{(4)}\right) P_{(n, m, p, q-3)}(x) \\
& +\sum_{k=0}^{q-4} P_{(n, m, p, k)}(x)\left(a_{(n, m, p, q-2)}^{(1)} \prod_{i=k+1}^{q-3}\left(b_{(n-1, m, p, i)}^{(1)}-b_{(n-1, m, p, i)}^{(4)}\right)\right. \\
& +a_{(n, m, p, q-2)}^{(2)} \prod_{i=k+1}^{q-3}\left(b_{(n, m-1, p, i)}^{(2)}-b_{(n, m-1, p, i)}^{(4)}\right) \\
& \left.+a_{(n, m, p, q-2)}^{(3)} \prod_{i=k+1}^{q-3}\left(b_{(n, m, p-1, i)}^{(3)}-b_{(n, m, p-1, i)}^{(4)}\right)\right) \\
& +P_{(n, m, p-1,0)}(x)\left(a_{(n, m, p, q-2)}^{(1)} \prod_{i=0}^{q-3}\left(b_{(n-1, m, p, i)}^{(1)}-b_{(n-1, m, p, i)}^{(4)}\right)\right. \\
& \quad+a_{(n, m, p, q-2)}^{(2)} \prod_{i=0}^{q-3}\left(b_{(n, m-1, p, i)}^{(2)}-b_{(n, m-1, p, i)}^{(4)}\right)
\end{aligned}
$$




$$
\begin{aligned}
& \left.+a_{(n, m, p, q-2)}^{(3)} \prod_{i=0}^{q-3}\left(b_{(n, m, p-1, i)}^{(3)}-b_{(n, m, p-1, i)}^{(4)}\right)\right) \\
& +\sum_{k=0}^{p-2} P_{(n, m, k, 0)}(x)\left(a_{(n, m, p, q-2)}^{(1)} \prod_{i=k+1}^{p-1}\left(b_{(n-1, m, i, 0)}^{(1)}-b_{(n-1, m, i, 0)}^{(3)}\right)\right. \\
& \times \prod_{i=0}^{q-3}\left(b_{(n-1, m, p, i)}^{(1)}-b_{(n-1, m, p, i)}^{(4)}\right) \\
& +a_{(n, m, p, q-2)}^{(2)} \prod_{i=k+1}^{p-1}\left(b_{(n, m-1, i, 0)}^{(2)}-b_{(n, m-1, i, 0)}^{(3)}\right) \\
& \left.\times \prod_{i=0}^{q-3}\left(b_{(n, m-1, p, i)}^{(2)}-b_{(n, m-1, p, i)}^{(4)}\right)\right) \\
& +P_{(n, m-1,0,0)}(x)\left(a_{(n, m, p, q-2)}^{(1)} \prod_{i=0}^{p-1}\left(b_{(n-1, m, i, 0)}^{(1)}-b_{(n-1, m, i, 0)}^{(3)}\right)\right. \\
& \times \prod_{i=0}^{q-3}\left(b_{(n-1, m, p, i)}^{(1)}-b_{(n-1, m, p, i)}^{(4)}\right) \\
& +a_{(n, m, p, q-2)}^{(2)} \prod_{i=0}^{p-1}\left(b_{(n, m-1, i, 0)}^{(2)}-b_{(n, m-1, i, 0)}^{(3)}\right) \\
& \left.\times \prod_{i=0}^{q-3}\left(b_{(n, m-1, p, i)}^{(2)}-b_{(n, m-1, p, i)}^{(4)}\right)\right) \\
& +\sum_{k=0}^{m-2} P_{(n, k, 0,0)}(x) a_{(n, m, p, q-2)}^{(1)} \prod_{i=k+1}^{m-1}\left(b_{(n-1, i, 0,0)}^{(1)}-b_{(n-1, i, 0,0)}^{(2)}\right) \\
& \times \prod_{i=0}^{p-1}\left(b_{(n-1, m, i, 0)}^{(1)}-b_{(n-1, m, i, 0)}^{(3)}\right) \\
& \times \prod_{i=0}^{q-3}\left(b_{(n-1, m, p, i)}^{(1)}-b_{(n-1, m, p, i)}^{(4)}\right) \\
& +P_{(n-1,0,0)}(x) a_{(n, m, p, q-2)}^{(1)} \prod_{i=0}^{m-1}\left(b_{(n-1, i, 0,0)}^{(1)}-b_{(n-1, i, 0,0)}^{(2)}\right) \\
& \times \prod_{i=0}^{p-1}\left(b_{(n-1, m, i, 0)}^{(1)}-b_{(n-1, m, i, 0)}^{(3)}\right) \\
& \times \prod_{i=0}^{q-3}\left(b_{(n-1, m, p, i)}^{(1)}-b_{(n-1, m, p, i)}^{(4)}\right) .
\end{aligned}
$$

By using (2.4) for $\left(n_{1}, n_{2}, n_{3}, n_{4}\right)=(n, m, p, q-1),(2.7)$, where $\ell=1$, for $\left(n_{1}, n_{2}, n_{3}, n_{4}\right)=$ $(n-1, m, p, q-2)$, (2.9), (2.7), where $\ell=2$, for $\left(n_{1}, n_{2}, n_{3}, n_{4}\right)=(n, m-1, p, q-2)$, (2.10) and (2.7), where $\ell=3$, for $\left(n_{1}, n_{2}, n_{3}, n_{4}\right)=(n, m, p-1, q-2)$, we obtain the last equation 


$$
\begin{aligned}
& x P_{(n, m, p, q-1)}(x) \\
& =P_{(n, m, p, q)}(x)+b_{(n, m, p, q-1)}^{(4)} P_{(n, m, p, q-1)}(x) \\
& +\left(a_{(n, m, p, q-1)}^{(1)}+a_{(n, m, p, q-1)}^{(2)}+a_{(n, m, p, q-1)}^{(3)}+a_{(n, m, p, q-1)}^{(4)}\right) P_{(n, m, p, q-2)}(x) \\
& +\sum_{k=0}^{q-3} P_{(n, m, p, k)}(x)\left(a_{(n, m, p, q-1)}^{(1)} \prod_{i=k+1}^{q-2}\left(b_{(n-1, m, p, i)}^{(1)}-b_{(n-1, m, p, i)}^{(4)}\right)\right. \\
& +a_{(n, m, p, q-1)}^{(2)} \prod_{i=k+1}^{q-2}\left(b_{(n, m-1, p, i)}^{(2)}-b_{(n, m-1, p, i)}^{(4)}\right) \\
& \left.+a_{(n, m, p, q-1)}^{(3)} \prod_{i=k+1}^{q-2}\left(b_{(n, m, p-1, i)}^{(3)}-b_{(n, m, p-1, i)}^{(4)}\right)\right) \\
& +P_{(n, m, p-1,0)}(x)\left(a_{(n, m, p, q-1)}^{(1)} \prod_{i=0}^{q-2}\left(b_{(n-1, m, p, i)}^{(1)}-b_{(n-1, m, p, i)}^{(4)}\right)\right. \\
& +a_{(n, m, p, q-1)}^{(2)} \prod_{i=0}^{q-2}\left(b_{(n, m-1, p, i)}^{(2)}-b_{(n, m-1, p, i)}^{(4)}\right) \\
& \left.+a_{(n, m, p, q-1)}^{(3)} \prod_{i=0}^{q-2}\left(b_{(n, m, p-1, i)}^{(3)}-b_{(n, m, p-1, i)}^{(4)}\right)\right) \\
& +\sum_{k=0}^{p-2} P_{(n, m, k, 0)}(x)\left(a_{(n, m, p, q-1)}^{(1)} \prod_{i=k+1}^{p-1}\left(b_{(n-1, m, i, 0)}^{(1)}-b_{(n-1, m, i, 0)}^{(3)}\right)\right. \\
& \times \prod_{i=0}^{q-2}\left(b_{(n-1, m, p, i)}^{(1)}-b_{(n-1, m, p, i)}^{(4)}\right) \\
& +a_{(n, m, p, q-1)}^{(2)} \prod_{i=k+1}^{p-1}\left(b_{(n, m-1, i, 0)}^{(2)}-b_{(n, m-1, i, 0)}^{(3)}\right) \\
& \left.\times \prod_{i=0}^{q-2}\left(b_{(n, m-1, p, i)}^{(2)}-b_{(n, m-1, p, i)}^{(4)}\right)\right) \\
& +P_{(n, m-1,0,0)}(x)\left(a_{(n, m, p, q-1)}^{(1)} \prod_{i=0}^{p-1}\left(b_{(n-1, m, i, 0)}^{(1)}-b_{(n-1, m, i, 0)}^{(3)}\right)\right. \\
& \times \prod_{i=0}^{q-2}\left(b_{(n-1, m, p, i)}^{(1)}-b_{(n-1, m, p, i)}^{(4)}\right) \\
& +a_{(n, m, p, q-1)}^{(2)} \prod_{i=0}^{p-1}\left(b_{(n, m-1, i, 0)}^{(2)}-b_{(n, m-1, i, 0)}^{(3)}\right) \\
& \left.\times \prod_{i=0}^{q-2}\left(b_{(n, m-1, p, i)}^{(2)}-b_{(n, m-1, p, i)}^{(4)}\right)\right) \\
& +\sum_{k=0}^{m-2} P_{(n, k, 0,0)}(x) a_{(n, m, p, q-1)}^{(1)} \prod_{i=k+1}^{m-1}\left(b_{(n-1, i, 0,0)}^{(1)}-b_{(n-1, i, 0,0)}^{(2)}\right)
\end{aligned}
$$




$$
\begin{aligned}
& \times \prod_{i=0}^{p-1}\left(b_{(n-1, m, i, 0)}^{(1)}-b_{(n-1, m, i, 0)}^{(3)}\right) \\
& \times \prod_{i=0}^{q-2}\left(b_{(n-1, m, p, i)}^{(1)}-b_{(n-1, m, p, i)}^{(4)}\right) \\
+P_{(n-1,0,0,0)}(x) a_{(n, m, p, q-1)}^{(1)} \prod_{i=0}^{m-1}\left(b_{(n-1, i, 0,0)}^{(1)}-b_{(n-1, i, 0,0)}^{(2)}\right) & \times \prod_{i=0}^{p-1}\left(b_{(n-1, m, i, 0)}^{(1)}-b_{(n-1, m, i, 0)}^{(3)}\right) \\
& \times \prod_{i=0}^{q-2}\left(b_{(n-1, m, p, i)}^{(1)}-b_{(n-1, m, p, i)}^{(4)}\right) .
\end{aligned}
$$

The derived equations can be written in the following way:

$$
x \mathbf{P}_{(n, m, p, q)}(x)=M_{(n, m, p, q)}^{(4)} \mathbf{P}_{(n, m, p, q)}(x)+P_{(n, m, p, q)}(x) \mathbf{e}_{(n, m, p, q)},
$$

where

$$
\mathbf{P}_{(n, m, p, q)}(x)=\left[\begin{array}{c}
P_{(0,0,0,0)}(x) \\
P_{(1,0,0,0)}(x) \\
\vdots \\
P_{(n, 0,0,0)}(x) \\
P_{(n, 1,0,0)}(x) \\
\vdots \\
P_{(n, m, 0,0)}(x) \\
P_{(n, m, 1,0)}(x) \\
\vdots \\
P_{(n, m, p, 0)}(x) \\
P_{(n, m, p, 1)}(x) \\
\vdots \\
P_{(n, m, p, q-1)}(x)
\end{array}\right], \quad \mathbf{e}_{(n, m, p, q)}=\left[\begin{array}{c}
0 \\
0 \\
\vdots \\
0 \\
0 \\
\vdots \\
0 \\
\vdots \\
0 \\
0 \\
\vdots \\
1
\end{array}\right]
$$

and $M_{(n, m, p, q)}^{(4)}$ is the following matrix of order $n+m+p+q$ :

$$
M_{(n, m, p, q)}^{(4)}=\left[\begin{array}{l}
A_{(n, m, p, q)}^{(1)} \\
A_{(n, m, p, q)}^{(2)} \\
A_{(n, m, p, q)}^{(3)} \\
A_{(n, m, p, q)}^{(4)}
\end{array}\right],
$$

where

$$
\begin{aligned}
& A_{(n, m, p, q)}^{(1)}=\left[\widetilde{A}_{(n, m, p, q)}^{(1)} \mid[0]_{n \times(m+p+q-1)}\right], \\
& A_{(n, m, p, q)}^{(2)}=\left[[0]_{m \times(n-1)}\left|\widetilde{A}_{(n, m, p, q)}^{(2)}\right|[0]_{m \times(p+q-1)}\right],
\end{aligned}
$$




$$
\begin{aligned}
A_{(n, m, p, q)}^{(3)}= & {\left.[0]_{p \times(n-1)}\left|\widetilde{A}_{(n, m, p, q)}^{(3)}\right|[0]_{p \times(q-1)}\right] } \\
A_{(n, m, p, q)}^{(4)}= & {\left[[0]_{q \times(n-1)} \mid \widetilde{A}_{(n, m, p, q)}^{(4)}\right] } \\
\widetilde{A}_{(n, m, p, q)}^{(1)}= & {\left[\begin{array}{ccccc}
b_{(0,0,0,0)}^{(1)} & 1 & & \\
a_{(1,0,0,0)}^{(1)} & b_{(1,0,0,0)}^{(1)} & 1 & & \\
& \ddots & \ddots & \ddots & \\
& & a_{(n-1,0,0,0)}^{(1)} & b_{(n-1,0,0,0)}^{(1)} & 1
\end{array}\right]_{n \times(n+1)}, }
\end{aligned}
$$

$$
\begin{aligned}
& \widetilde{A}_{(n, m, p, q)}^{(2)}= \\
& {\left[\begin{array}{ccccccc}
a_{(n, 0,0,0)}^{(1)} & b_{(n, 0,0,0)}^{(2)} & 1 & & & & \\
a_{(n, 1,0,0)}^{(1)} \Pi_{0,0}^{(1-2)} & a_{(n, 1,0,0)}^{(1)}+a_{(n, 1,0,0)}^{(2)} & b_{(n, 1,0,0)}^{(2)} & 1 & & & \\
\vdots & \vdots & & \ddots & \ddots & & \\
a_{(n, m-2,0,0)}^{(1)} \Pi_{0, m-3}^{(1-2)} & a_{(n, m-2,0,0)}^{(1)} \Pi_{1, m-3}^{(1-2)} & \ldots & \ldots & & 1 & \\
a_{(n, m-1,0,0)}^{(1)} \Pi_{0, m-2}^{(1-2)} & a_{(n, m-1,0,0)}^{(1)} \Pi_{1, m-2}^{(1-2)} & \cdots & \cdots & & b_{(n, m-1,0,0)}^{(2)} & 1
\end{array}\right]_{m \times(m+2)}} \\
& \left.\begin{array}{l}
\widetilde{A}_{(n, m, p, q)}^{(3)} \\
\widetilde{A}_{(n, m, p, q)}^{(4)}
\end{array}\right\} \text { see Table 2.1. }
\end{aligned}
$$

Here we used the notation

$$
\begin{array}{ll}
\Pi_{k, \ell}^{(1-2)}=\prod_{i=k}^{\ell}\left(b_{(n-1, i, 0,0)}^{(1)}-b_{(n-1, i, 0,0)}^{(2)}\right), & \Pi_{k, \ell}^{(1-3)}=\prod_{i=k}^{\ell}\left(b_{(n-1, m, i, 0)}^{(1)}-b_{(n-1, m, i, 0)}^{(3)}\right) \\
\Pi_{k, \ell}^{(2-3)}=\prod_{i=k}^{\ell}\left(b_{(n, m-1, i, 0)}^{(2)}-b_{(n, m-1, i, 0)}^{(3)}\right), & \Pi_{k, \ell}^{(1-4)}=\prod_{i=k}^{\ell}\left(b_{(n-1, m, p, i)}^{(1)}-b_{(n-1, m, p, i)}^{(4)}\right), \\
\Pi_{k, \ell}^{(2-4)}=\prod_{i=k}^{\ell}\left(b_{(n, m-1, p, i)}^{(2)}-b_{(n, m-1, p, i)}^{(4)}\right), & \Pi_{k, \ell}^{(3-4)}=\prod_{i=k}^{\ell}\left(b_{(n, m, p-1, i)}^{(3)}-b_{(n, m, p-1, i)}^{(4)}\right) .
\end{array}
$$

For an arbitrary number $r>4$, one has $r-1$ lemmas which are generalization of the Lemmas 2.1-2.3. Each lemma provides a connection between $P_{\mathbf{n}+\mathbf{e}_{k}}(x)$ and $P_{\mathbf{n}+\mathbf{e}_{j}}(x)$, $j=1,2, \ldots, k-1, k=2,3, \ldots, r$. The first part of the construction of the type II multiple orthogonal polynomial $P_{\mathbf{n}}(x)$ is the same as in the case $r=4$, i.e., one obtains the polynomial $P_{\left(n_{1}, n_{2}, n_{3}, n_{4}, 0, \ldots, 0\right)}(x)$. After that, following a similar procedure, one can obtain $P_{\left(n_{1}, n_{2}, n_{3}, n_{4}, n_{5}, 0 \ldots, 0\right)}(x), \ldots, P_{\mathbf{n}}(x)$. The main problem in the construction of type II multiple orthogonal polynomials for $r>4$ is the notation since for larger $r$, the corresponding formulae and matrices are definitely even more complicated.

3. Optimal set of quadrature rules. Starting with a problem that arises in the evaluation of computer graphics illumination models, Borges [5] has examined the problem of numerically evaluating a set of $r \in \mathbb{N}, r \geq 2$, definite integrals with the same integrand and over the same interval of integration but related to different weight functions. Since for such integrals it is not efficient to use a set of $r$ Gauss-Christoffel quadrature rules, the problem of finding 


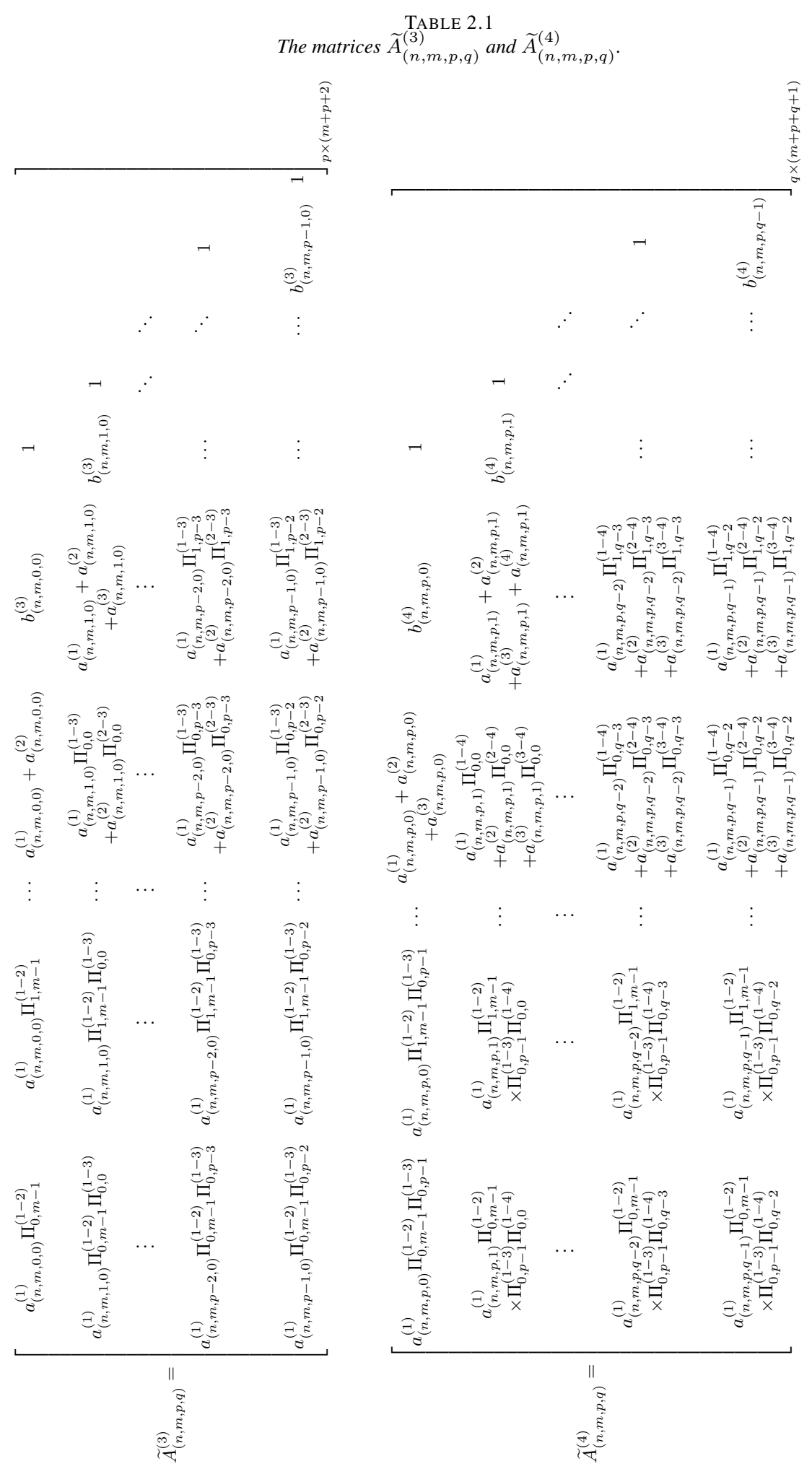


appropriate quadrature rules was studied in $[1,5,6,7,14]$. The solution of that problem given in $[14,15,19]$ introduces optimal sets of quadrature formulas.

DEFINITION 3.1. Let $W=\left(w_{1}, w_{2}, \ldots, w_{r}\right)$ be an AT system on an interval $E$, and let $\mathbf{n}=\left(n_{1}, n_{2}, \ldots, n_{r}\right)$ be a multi-index. A set of quadrature rules of the form:

$$
\int_{E} f(x) w_{k}(x) \mathrm{d} x \approx \sum_{i=1}^{|\mathbf{n}|} A_{k, i} f\left(x_{i}\right), \quad k=1,2, \ldots, r,
$$

will be called an optimal set in the sense of Borges with respect to $(W, \mathbf{n})$ if and only if the weight coefficients $A_{k, i}, k=1,2, \ldots, r, i=1,2, \ldots,|\mathbf{n}|$, and the nodes $x_{i}, i=1,2, \ldots,|\mathbf{n}|$, satisfy the following equations:

$$
\begin{aligned}
\sum_{i=1}^{|\mathbf{n}|} A_{k, i} & =\int_{E} w_{k}(x) \mathrm{d} x \\
\sum_{i=1}^{|\mathbf{n}|} A_{k, i} x_{i} & =\int_{E} x w_{k}(x) \mathrm{d} x \\
& \vdots \\
\sum_{i=1}^{|\mathbf{n}|} A_{k, i} x_{i}^{|\mathbf{n}|+n_{k}-1} & =\int_{E} x^{|\mathbf{n}|+n_{k}-1} w_{k}(x) \mathrm{d} x
\end{aligned}
$$

for $k=1,2, \ldots, r$.

In order to generalize the fundamental theorem of Gauss-Christoffel quadrature rules, the following theorem (see [14]) gives a characterization of the optimal set of quadrature rules by connecting them with the type II multiple orthogonal polynomials.

THEOREM 3.2. Let $\mathbf{n}$ be a multi-index, and let $W=\left(w_{1}, w_{2}, \ldots, w_{r}\right)$ be an AT system on an interval E. A set of quadrature rules (3.1) is the optimal set in the sense of Borges with respect to $(W, \mathbf{n})$ if and only if:

(i) all rules are exact for all polynomials of degree less than or equal to $|\mathbf{n}|-1$,

(ii) the polynomial $q(x)=\prod_{i=1}^{|\mathbf{n}|}\left(x-x_{i}\right)$ is the type II multiple orthogonal polynomial $P_{\mathbf{n}}(x)$ with respect to $(W, \mathbf{n})$.

REMARK 3.3. According to Theorem 1.1, all zeros of the type II multiple orthogonal polynomial $P_{\mathbf{n}}(x)$ with respect to $(W, \mathbf{n})$ are simple and lie on the interval $E$.

In the case of the famous Gauss-Christoffel quadrature rule $(r=1)$, the nodes and the weights can be expressed in terms of an eigenvalue problem of a Jacobi matrix containing the recurrence coefficients (see, for example, [9, Chapter 3, Section 2.3]). In order to extend this to the optimal set of quadrature rules, we will use the square matrices that have been obtained in Section 2, whose eigenvalues are nodes of this optimal set of quadrature rules. Then the weights can be calculated by solving systems of linear equations. The corresponding method for calculating the nodes and weight coefficients of the optimal set of quadrature rules with respect to a nearly-diagonal multi-index was given in [14]. In [19], the cases of $r=2$ and $r=3$ weight functions are explained in detail. Here we give a construction of the optimal set of quadrature rules for $r=4$ weight functions. In a similar way the optimal set of quadrature rules for arbitrary $r>4$ can be constructed.

Let $x_{i}=x_{i}^{(n, m, p, q)}, i=1,2, \ldots, n+m+p+q$, be the zeros of $P_{(n, m, p, q)}(x)$. Then (2.11) reduces to the following eigenvalue problem:

$$
x_{i} \mathbf{P}_{(n, m, p, q)}\left(x_{i}\right)=M_{(n, m, p, q)}^{(4)} \mathbf{P}_{(n, m, p, q)}\left(x_{i}\right) .
$$


Thus, the zeros of $P_{(n, m, p, q)}(x)$, i.e., the nodes of the optimal set of quadrature rules (3.1), are the eigenvalues of the matrix $M_{(n, m, p, q)}$.

Let

$$
V_{(n, m, p, q)}=\left[\begin{array}{llll}
\mathbf{P}_{(n, m, p, q)}\left(x_{1}\right) & \mathbf{P}_{(n, m, p, q)}\left(x_{2}\right) & \cdots & \mathbf{P}_{(n, m, p, q)}\left(x_{n+m+p+q}\right)
\end{array}\right]
$$

denote the matrix of the eigenvectors of the matrix $M_{(n, m, p, q)}^{(4)}$, each normalized so that the first component is equal to 1 . Then the weight coefficients $A_{k, i}, k=1,2, \ldots, r, i=1,2, \ldots, n+$ $m+p+q$, can be obtained by solving the following systems of linear equations

$$
V_{(n, m, p, q)}\left[\begin{array}{c}
A_{k, 1} \\
A_{k, 2}^{*(k)} \\
\vdots \\
A_{k, n+m+p+q}
\end{array}\right]=\left[\begin{array}{c}
(0,0,0,0) \\
\mu_{(1,0,0,0)}^{*(k)} \\
\vdots \\
\mu_{(n, 0,0,0)}^{*(k)} \\
\mu_{(n, 1,0,0)}^{*(k)} \\
\vdots \\
\mu_{(n, m, 0,0)}^{*(k)} \\
\vdots \\
\mu_{(n, m, p, 0)}^{*(k)} \\
\vdots \\
\mu_{(n, m, p, q-1)}^{*(k)}
\end{array}\right], \quad k=1,2, \ldots, r
$$

where

$$
\mu_{(i, j, \ell, t)}^{*(k)}=\int_{E} P_{(i, j, \ell, t)}(x) w_{k}(x) \mathrm{d} x
$$

$i=0,1, \ldots, n, j=0,1, \ldots, m, \ell=0,1, \ldots, p, t=0,1, \ldots, q-1, k=1,2, \ldots, r$, and each rule exactly integrates the modified moments.

3.1. Numerical construction for $r=4$. In this section we present an effective numerical method for constructing the optimal set of quadrature rules. For that purpose we construct the matrix $M_{(n, m, p, q)}^{(4)}$ and the corresponding type II multiple orthogonal polynomial $P_{(n, m, p, q)}(x)$.

In order to obtain the nearest neighbor recurrence coefficients (1.4), i.e., the elements of the matrix $M_{(n, m, p, q)}^{(4)}$, we start by calculating the corresponding coefficients of the marginal (1.2) or nearly-diagonal recurrence relations (1.3), which can be obtained by using the discretized Stieltjes-Gautschi procedure (see [10] and [14]). Now, the nearest neighbor recurrence coefficients can be obtained in two ways. The first one is to start with the coefficients of the marginal recurrence relations and use [8, Theorem 3.1], and in the second we start with the coefficients of the nearly-diagonal recurrence relations and use [8, Theorem 2.3]. The nodes of the optimal set of quadrature rules (3.1) are the eigenvalues of the matrix $M_{(n, m, p, q)}^{(4)}$. By using the method given in Section 2, the type II multiple orthogonal polynomial $P_{(n, m, p, q)}(x)$ can be obtained. Finally, by solving the system (3.2) one can find the weight coefficients of the optimal set of quadrature rules (3.1). This construction is illustrated by the following example.

EXAMPLE 3.4. Let us construct the optimal set of quadrature rules with respect to the weight functions $W=\left(w_{1}, w_{2}, w_{3}, w_{4}\right)$, where $w_{1}=(1-x)(1+x)^{\frac{1}{2}}, w_{2}=(1-x)(1+x)^{-\frac{1}{4}}$, 


\section{ETNA}

Kent State University and

Johann Radon Institute (RICAM)

$w_{3}=(1-x)(1+x)^{\frac{1}{4}}, w_{4}=(1-x)(1+x)^{\frac{3}{4}}, x \in(-1,1)$, and the multi-index $\mathbf{n}=(2,1,2,1)$. The results are given in Table 3.1.

TABLE 3.1

The nodes $x_{i}$ and the weight coefficients $A_{k, i}, k=1,2,3,4, i=1,2, \ldots, 6$, of the optimal set of quadrature rules with respect to $W=\left((1-x)(1+x)^{\frac{1}{2}},(1-x)(1+x)^{-\frac{1}{4}},(1-x)(1+x)^{\frac{1}{4}},(1-x)(1+x)^{\frac{3}{4}}\right), x \in(-1,1)$, and $\mathbf{n}=(2,1,2,1)$.

\begin{tabular}{c|r|l|l|l|l}
$i$ & \multicolumn{1}{|c|}{$x_{i}$} & \multicolumn{1}{c|}{$A_{1, i}$} & \multicolumn{1}{|c}{$A_{2, i}$} & \multicolumn{1}{|c}{$A_{3, i}$} & \multicolumn{1}{|c}{$A_{4, i}$} \\
\hline 1 & -0.98475327357 & 0.0127814715512 & 0.29556494481 & 0.035780213859 & 0.0045063978573 \\
2 & -0.85938607140 & 0.14656451060 & 0.63851330264 & 0.23941347025 & 0.089783863948 \\
3 & -0.55789207493 & 0.40180768546 & 0.74106392071 & 0.49276056219 & 0.32763023233 \\
4 & -0.10715042849 & 0.51883443313 & 0.56486887209 & 0.53374419313 & 0.50434293040 \\
5 & 0.39114058354 & 0.34259892476 & 0.26745887246 & 0.31545961942 & 0.37207289191 \\
6 & 0.80082931606 & 0.085907441037 & 0.055262019488 & 0.074158726943 & 0.099517464759
\end{tabular}

\section{REFERENCES}

[1] A. Angelesco, Sur l'approximation simultanée de plusieurs intégrals définies, C. R. Acad. Sci. Paris, 167 (1918), pp. 629-631.

[2] A. Aptekarev, Multiple orthogonal polynomials, J. Comput. Appl. Math., 99 (1998), pp. $423-447$.

[3] A. I. Aptekarev, A. Branquinho, And W. VAn Assche, Multiple orthogonal polynomials for classical weights, Trans. Amer. Math. Soc., 355 (2003), pp. 3887-3914.

[4] B. Beckermann, J. Coussement, And W. Van Assche, Multiple Wilson and Jacobi-Piñeiro polynomials, J. Approx. Theory, 132 (2005), pp. 155-181.

[5] C. F. Borges, On a class of Gauss-like quadrature rules, Numer. Math., 67 (1994), pp. 271-288.

[6] J. CoussEment AND W. VAN Assche, Gaussian quadrature for multiple orthogonal polynomials, J. Comput. Appl. Math., 178 (2005), pp. 131-145.

[7] U. Fidalgo Prieto, J. Illán, AND G. LóPez Lagomasino, Hermite-Padé approximation and simultaneous quadrature formulas, J. Approx. Theory, 126 (2004), pp. 171-197.

[8] G. FiliPUK, M. HANECZOK, AND W. VAN AsSCHE, Computing recurrence coefficients of multiple orthogonal polynomials, Numer. Algorithms, 70 (2015), pp. 519-543.

[9] W. GAUTSCHI, Numerical Analysis. An Introduction, Birkhäuser, Boston, 1997.

[10] - Orthogonal polynomials: applications and computation, Acta Numerica, 5 (1996), pp. 45-119.

[11] J. S. GERONImo, P. ILIEV, AND W. VAN Assche, Alpert multiwavelets and Legendre-Angelesco multiple orthogonal polynomials, SIAM J. Math. Anal., 49 (2017), pp. 626-645.

[12] M. E. H. IsmaIL, Classical and Quantum Orthogonal Polynomials in One Variable, Encyclopedia of Mathematics and its Applications 98, Cambridge University Press, Cambridge 2005.

[13] D. W. LEE, Classical multiple orthogonal polynomials of Angelesco system, Appl. Numer. Math., 60 (2010), pp. 1342-1351.

[14] G. Milovanović And M. Stanić, Construction of multiple orthogonal polynomials by discretized StieltjesGautschi procedure and corresponding Gaussian quadratures, Facta Univ. Ser. Math. Inform., 18 (2003), pp. 9-29.

[15] - Multiple orthogonality and applications in numerical integration, in Nonlinear Analysis: Stability, Approximation, and Inequalities, P. M. Pardalos, P. Georgiev, and H. M. Srivastava, eds., Springer Optimization and its Applications, 68, Springer, New York, 2012, pp. 431-455.

[16] T. Neuschel AND W. VAN Assche, Asymptotic zero distribution of Jacobi-Piñeiro and multiple Laguerre polynomials, J. Approx. Theory, 205 (2016), pp. 114-132.

[17] E. M. Nikishin AND V. N. SoRoKin, Rational Approximations and Orthogonality, Translations of Mathematical Monographs 92, Amer. Math. Soc., Providence,1991.

[18] V. N. SOROKIn, Generalization of classical polynomials and convergence of simultaneous Padé approximants, Trudy Sem. Petrovsk., 11 (1986), pp. 125-165

[19] T. V. Tomović AND M. P. STANiĆ, Construction of the optimal set of two or three quadrature rules in the sense of Borges, Numer. Algorithms, 78 (2018), pp. 1087-1109.

[20] W. Van Assche AND E. Coussement, Some classical multiple orthogonal polynomials, J. Comput. Appl. Math., 127 (2001), pp. 317-347.

[21] W. VAN ASSCHE, Multiple orthogonal polynomials, irrationality and transcendence, in Continued Fractions: From Analytic Number Theory to Constructive Approximation, B. C. Berndt and F. Gesztesy, eds., Contemp. 
ETNA

Kent State University and

Johann Radon Institute (RICAM)

Math. 236, Amer. Math. Soc., Providence, 1999, pp. 325-342.

[22] - Nearest neighbor recurrence relations for multiple orthogonal polynomials, J. Approx. Theory, 163 (2011), pp. 1427-1448.

[23] - Nonsymmetric linear difference equations for multiple orthogonal polynomials, in SIDE IIIsymmetries and integrability of difference equations, D. Levi and O. Ragnisco, eds., CRM Proceedings and Lecture Notes 25, Amer. Math. Soc., Providence, 2000, pp. 415-429, 